\title{
Research and Comparison on the Method of Determining the Nonlinearity of Univariate Function
}

\author{
Qingbin ZHANG, Wei SHEN, Wenliang FAN, Xiaomeng XIE \\ School of Civil Engineering \\ Chongqing University \\ Chongqing, China \\ e-mail: 1668677065@qq.com
}

\begin{abstract}
In the analysis of stochastic system, the nonlinearity of function has an important influence on the accuracy and efficiency, but the corresponding researches are very scarce. In this paper, two kinds of methods are put forward to determine the nonlinearity of the univariate function. Firstly, the nonlinearity of the univariate function is defined based on the difference of numerical integration; secondly, the first method gets the nonlinearity of the univariate function via the number of the computing nodes based on point estimate while it is determined by the order of the response function based on orthogonal polynomial in the second method. Finally, the two methods were compared by a number of examples, the results show that both of the two methods can accurately get the nonlinearity of the univariate function, the first method is easier to get while the second method has higher efficiency.
\end{abstract}

Keywords-stochastic analysis; nonlinearity; univariate function; point estimate; orthogonal polynomial

\section{INTRODUCTION}

The nonlinearity is a very important characteristics of the function, which is the foundation of stochastic analysis. If the nonlinearity of a function can be determined in advance, the efficiency will be highly improved in many stochastic problems. Such as in point estimate method, which is one of the simplest and most efficient approaches for calculating probability moments and the main topics for the analysis of stochastic function or system. According to the way of determining the number of nodes, the point estimation method can be divided into two categories. One is using the fixed number of nodes, for example, the PEM proposed by Rosenblueth [1], [2] needs $2^{n}$ nodes for function or system of $n$-dimension dependent variables; Harr's method[3] and a modified Harr's algorithm[4] reduce the nodes from $2^{n}$ to $2 n$; Li's method[5] requires $\left(n^{2}+3 n+2\right) / 2$ nodes. Another is using the unfixed number of nodes, such as Zhao \& Ono's method[6], [7], Nataf transform method based on point estimation[8], all of them require $n \times m+1$ nodes, where $m$ is the number of nodes used in the point estimates for functions of single random variables, and is always determined subjectively and empirically.

The paper is organized as follows: in Section 2, two kinds of methods have been proposed to calculate the nonlinearity of the univariate function, One is the adaptive iterative method (AIM), in which the algebraic nonlinearity for function of single random variables is deduced, and then the number of necessary points is determined rationally, the other is the orthogonal polynomial fitting method(OPFM), which calculate the nonlinearity by the order of the response function based on orthogonal polynomial. In Section 3, an example is numerically examined to verify the accuracy and efficiency of the proposed methods, Finally, Section 4 summaries some conclusions.

\section{Methods OF DETERMINING THE NONLINEARITY OF UNIVARIATE FUNCTION}

Considered a general function of a single variable, that is

$$
Z=g(X)
$$

where $\mathrm{Z}$ is the system response, $\mathrm{X}$ is the variable, and $\mathrm{g}($.$) is$ a general function describing the relation between $\mathrm{X}$ and $\mathrm{Z}$. when $X$ is not the normal variable, the Rosenblatt transformation can be exploited to transform it into the standard normal variable.

Therefore, Eq.(1) can be expressed by

$$
Z=g\left(R^{-1}(U)\right)=h(U)
$$

where $R^{-1}$ is the inverse of Rosenblatt transformation.

It is well known that the nonlinearity of $h(U)$ is not determined at first and lots of iterative are needed to ensure algebraic accuracy, which is always inefficient. So there are two kind of methods proposed to improve the efficiency. In this paper, the nonlinearity of univariate function is defined as the algebraic precision which is necessary to obtain the accurate result of numerical integration as the algebra nonlinearity of the function.

\section{A. Adaptive Iterative Method}

1) Criterion for judging the algebra nonlinearity of the function

Based on Eq. (2), calculation of probability moments for $\mathrm{Z}$ can be expressed as

$$
\begin{gathered}
\mu_{z}=M_{z, 1}=E[h(U)] \approx \sum_{i=1}^{m} P_{i} \cdot h\left(u_{i}\right) \\
M_{z, k}=E\left[\left(h(U)-\mu_{z}\right)^{k}\right] \approx \sum_{i=1}^{m} P_{i} \cdot\left[h\left(u_{\mathrm{i}}\right)-\mu_{z}\right]^{k}
\end{gathered}
$$


where $m$ is the number of nodes determined empirically; $\mu_{Z}$ is the mean value of $\boldsymbol{Z} ; M_{Z, k}$ is the $k$ th central moment of $\boldsymbol{Z}$, in order to make the further elaboration convenient, $\mu_{Z}$ be written as $M_{Z, 1}$ in this paper; the node $u_{i}$ and corresponding weight $P_{i}$ for a standard normal random variable can be obtained by utilizing those of Hermite integration, that is $u_{i}=\sqrt{2} y_{i}, P_{i}=\omega_{i} / \sqrt{\pi}$, in which $y_{i}$ and $\omega_{i}$ denote nodes and corresponding weights of Hermite integration, respectively, obtained by consulting mathematics handbook or calculating as reference do. Observing Eq. (4), it is easy to find that the integral algebra nonlinearity in $M_{Z, q}$ is $q$ times than that of $M_{Z, 1}$ and $q / k$ times than $M_{Z, k}$, therefore, the degree of nonlinear for function $h(U)$ can be deduced via the iterative convergence criterion of lower statistical moments, and then the number of necessary points is determined by calculating the algebra nonlinearity of $q$ statistical moments. If $m_{j 1}$ nodes and $m_{j 2}$ nodes are used to calculate $M_{Z, k}$ respectively, and

$$
\left|\left(M_{Z, k, m_{j 2}}-M_{Z, k, m_{j 1}}\right) / M_{Z, k, m_{j 2}}\right|<\varepsilon
$$

then it can be considered that the algebra nonlinearity of $h^{k}(U)$ is approximately on more than $2 \times m_{j 1}-1$, correspondingly, the algebra nonlinearity of $h(U)$ should be approximately on more than ceiling $\left[\left(2 m_{j 1}-1\right) / k\right]$, and that of integral in $M_{Z, q}$ is approximately no more than ceiling $\left[q \cdot\left(2 m_{j 1}-1\right) / k\right]$, where ceiling $[\cdot]$ denotes the minimal integer which is greater than or equal to the independent variable. As is known to all, the lower the order of probability moments are, the less nodes are required theoretically. Therefore, in order to improve the efficiency of judgment for the integral algebra nonlinearity in $M_{Z, q}$, usually $k=1$ in Eq. (5), that is to say, $\mu_{Z}$ is used for judging algebra nonlinearity.

\section{2) Realization of adaptive iterative method}

Based on the criterion for judging the algebra nonlinearity of the function of single random variables, adaptive iterative method can be realized as following steps:

- Select $\mathrm{m}=1,3$, calculate $\mathrm{Mz}, 1,1$ and $\mathrm{Mz}, 1,3$ then compare them, if

$$
\left|\left(M_{z, 1,3}-M_{z, 1,1}\right) / M_{z, 1,3}\right|<\varepsilon
$$

- then $\mathrm{k}=2$, or $\mathrm{k}=1$, where $\varepsilon=5.0 \times 10-3$;

If $\mathrm{k}=1$, select $\mathrm{m}=2$, calculate $\mathrm{Mz}, 1,2$, If

$$
\left|\left(M_{z, 1,3}-M_{z, 1,2}\right) / M_{z, 1,3}\right|<\varepsilon
$$

- the nonlinearity of $\mathrm{g}(\mathrm{X})$ is 3 , and the algebraic accuracy of the Mz,q is $3 \mathrm{q}$; or let $\mathrm{m} 3=3, \mathrm{j}=4$, perform the following steps:

- (a) select $\mathrm{m}=\mathrm{mj}=\mathrm{mj}-1+\mathrm{k}$, calculate $\mathrm{Mz}, \mathrm{k}, \mathrm{mj}$;

- (b) If

$$
\left|\left(M_{z, k, m_{j}}-M_{z, k, m_{j-1}}\right) / M_{z, k, m_{j}}\right|<\varepsilon
$$

the nonlinearity of $\mathrm{g}(X)$ is ceiling $\left[\left(2 m_{\mathrm{j}-1}-1\right) / k\right]$; or let $j=j+1$, turn to a).

- If $\mathrm{k}=2$, then calculate $\mathrm{MZ}, \mathrm{k}, 3$, and let $\mathrm{m} 2=3, \mathrm{j}=3$, perform the following steps:

- Select $m=m j=m j-1+k$, calculate $M Z, k, m j$;

- If equation (8) run, and the nonlinearity of $g(X)$ is ceiling[(2mj-1-1)/k]; or let $\mathrm{j}=\mathrm{j}+1$, turn to( a).

\section{B. Orthogonal Polynomial Fitting Method}

1) Criterion for judging the algebra nonlinearity of the function

Orthogonal polynomial is a practical approach in the analysis of stochastic system, in this section, the nonlinearity of the univariate function is estimated by the required order of orthogonal polynomial based on orthogonal polynomial fitting, and the Hermite orthogonal polynomials are shown in Table1.

The univariate function can be approximated by

$$
h_{i}^{\left(m_{i}\right)}\left(u_{i}\right)=\sum_{j=0}^{m_{i}} \lambda_{i j} \psi_{j}\left(u_{i}\right)
$$

where $m_{i}$ is the order of the orthogonal polynomial $\psi_{j}\left(u_{i}\right), \lambda_{i j}$ is the coefficient and

$$
\lambda_{i j}=\frac{E\left[h_{i}\left(u_{i}\right) \psi_{j}\left(u_{i}\right)\right]}{E\left[\left[\psi_{j}\left(u_{i}\right)\right]^{2}\right]}=\frac{\sum_{k=1}^{n_{0}} w_{i, k} \cdot h_{i}\left(u_{i, k}\right) \psi_{j}\left(u_{i, k}\right)}{\sum_{k=1}^{n_{0}} w_{i, k} \cdot\left[\psi_{j}\left(u_{i, k}\right)\right]^{2}}
$$

where $\mathrm{E}[\cdot]$ is the expectation operator, $u_{i, k}$ and $w_{i, k}$ are the abscissas(in ascending order) and weights of the Gauss-Hermite quadrature formula, then calculate the standard deviation of $h_{i}\left(x_{i}\right)$ and $h_{i}^{\left(m_{i}\right)}\left(u_{i}\right)$ as follows:

$$
\mu_{h_{i}}=E\left[h_{i}\left(u_{i}\right)\right] \approx \sum_{k=1}^{n_{0}} w_{i, k} \cdot h_{i}\left(u_{i, k}\right)
$$

$$
\begin{aligned}
\sigma_{h_{i}} & =\sqrt{E\left[\left(h_{i}\left(u_{i}\right)-\mu_{h_{i}}\right)^{2}\right]} \\
& \approx \sqrt{\sum_{k=1}^{n_{0}} w_{i, k} \cdot\left[h_{i}\left(u_{i, k}\right)-\mu_{h_{i}}\right]^{2}}
\end{aligned}
$$

$$
\mu_{h_{i}^{\left(m_{i}\right)}}=E\left[h_{i}^{\left(m_{i}\right)}\left(u_{i}\right)\right] \approx \sum_{k=1}^{n_{0}} w_{i, k} \cdot h_{i}^{\left(m_{i}\right)}\left(u_{i, k}\right)
$$




$$
\begin{aligned}
\sigma_{h_{i}^{\left(m_{i}\right)}} & =\sqrt{E\left[\left(h_{i}^{\left(m_{i}\right)}\left(u_{i}\right)-\mu_{h_{i}^{\left(m_{i}\right)}}\right)^{2}\right]} \\
& \approx \sqrt{\sum_{k=1}^{n_{0}} w_{i, k} \cdot\left[h_{i}^{\left(m_{i}\right)}\left(u_{i, k}\right)-\mu_{h_{i}^{\left(m_{i}\right)}}\right]^{2}}
\end{aligned}
$$

then compare the standard deviation, if

$$
\frac{\sigma_{h_{i}^{\left(m_{i}\right)}}-\sigma_{h_{i}}}{\sigma_{h_{i}}}<\varepsilon
$$

the nonlinearity of $x_{i}$ is $m_{i}$, where $\varepsilon=5.0 \times 10^{-3}$.

TABLE I. HERMITE ORTHOGONAL POLYNOMIALS

\begin{tabular}{|l|l|}
\hline Variables & Standard normal \\
\hline Support & {$[-\infty,+\infty]$} \\
\hline Probability density & $\exp \left(-u^{2} / 2\right)$ \\
\hline Weight function & $\exp \left(-u^{2} / 2\right)$ \\
\hline \multirow{3}{*}{ First four polynomials } & $\psi_{0}=1$ \\
& $\begin{array}{l}\psi_{1}(u)=u \\
\psi_{2}(u)=u^{2}-1 \\
\psi_{3}(u)=u^{3}-3 u\end{array}$ \\
\hline Recurrence relation & $\psi_{i+1}(u)=u \psi_{i}(u)-i \psi_{i-1}(u)$ \\
\hline
\end{tabular}

2) Realization of Orthogonal Polynomial Fitting Method

Based on the orthogonal polynomial fitting method, the procedures are

- Select $\mathrm{n} 0=7$, calculate the standard deviation of the univariate component function by Eqs.(11) and (12).

- Calculate coefficients with $\mathrm{n} 0=7$ by Eq. (10).

- Calculate the standard deviation of hi(mi)(xi) by Eqs. (13) and (14).

- Compare the standard deviation of hi(mi) (ui) and hi (ui), if Eq. (15) run, then turn to step e), or let $\mathrm{mi}=\mathrm{mi}+1$, turn back to step $\mathrm{c}$ ).

- If $m i \leq n 0$, then $m=m i, n=n 0$, the nonlinearity of the function is $\mathrm{m}$; or $\mathrm{n} 0=\mathrm{n} 0+4$, turn back to step a).

\section{NUMERICAL EXAMPLES}

Consider a univariate polynomial function

$$
Y=2 X^{k}+3
$$

where $\mathrm{k}=3, \ldots, 6$, respectively, $\mathrm{X}$ are standard normal random variable, lognormal random variable with mean value 5 and coefficient of variation 0.2 and extreme type I distributed random variable, respectively, so 12 sub examples are included. For each sub example, calculate the nonlinearity through AIM (adaptive iterative method) and OPFM (Orthogonal polynomial fitting method), respectively, and compare the results. Table 2 shows the nonlinearity of different methods varied with $\mathrm{k}$ and variables while Table 3 shows the necessary number of function calls for different methods (for the engineering structure, denoting analysis times).
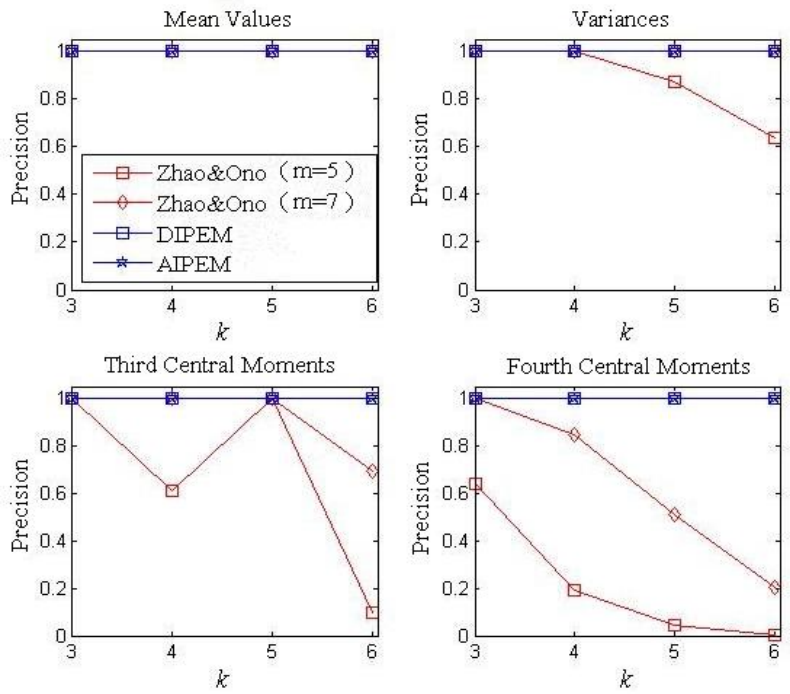

Figure 1. Trend of precision for different methods varied with $k$ as $X$ is standard normal random variable.
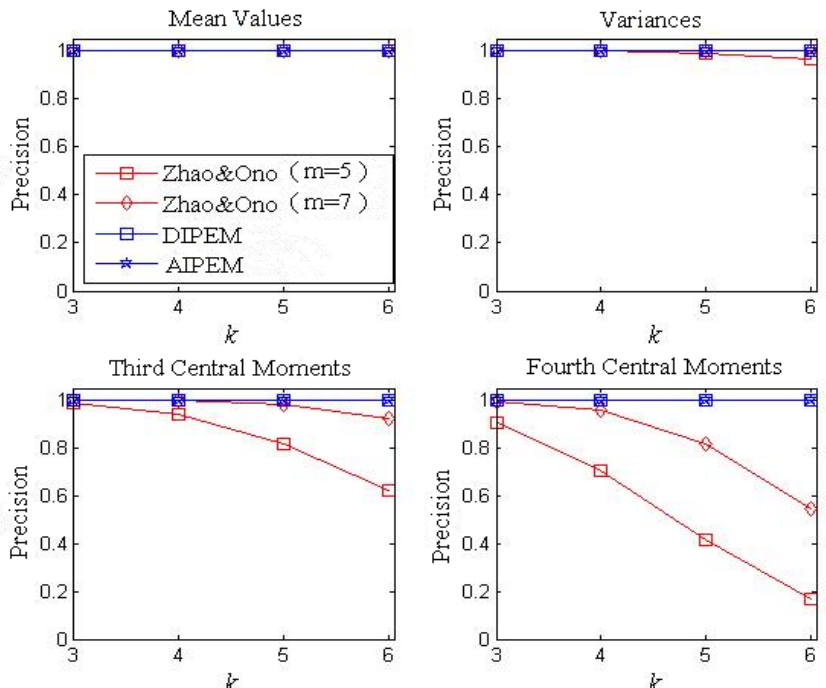

Figure 2. Trend of precision for different methods varied with $\mathrm{k}$ as $\mathrm{X}$ is lognormal random variable. 

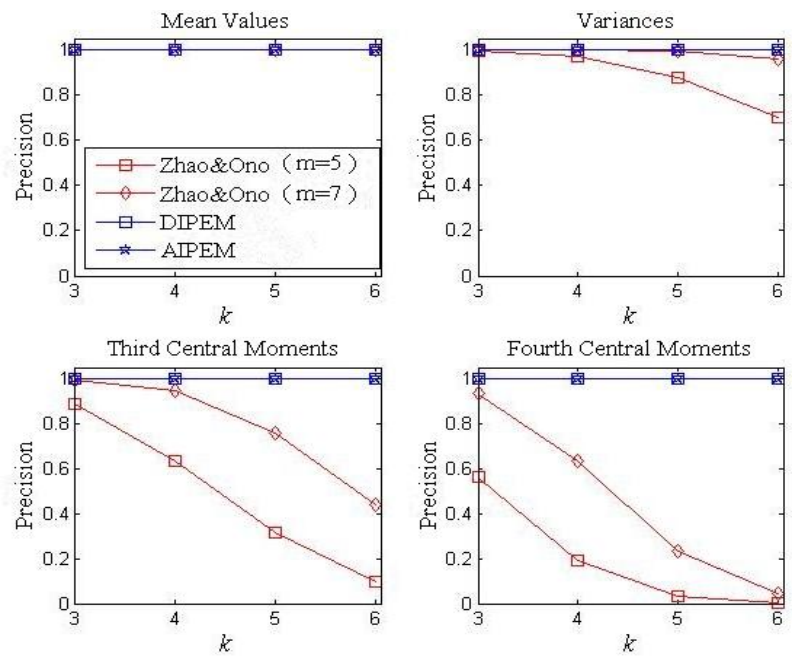

Figure 3. Trend of precision for different methods varied with $\mathrm{k}$ as $\mathrm{X}$ is extreme type I random variable.

TABLE II. THE RESULTS OF THE TWO METHODS

\begin{tabular}{llllll}
\hline$k$ & & 3 & 4 & 5 & 6 \\
\hline \multirow{2}{*}{ Standard normal } & AIM & 3 & 5 & 5 & 7 \\
\multirow{2}{*}{ Lognormal } & OPFM & 3 & 4 & 5 & 6 \\
\multirow{2}{*}{ Extreme Type I } & AIM & 4 & 6 & 6 & 8 \\
& OPFM & 4 & 5 & 6 & 7 \\
& AIM & 4 & 7 & 7 & 8 \\
& OPFM & 4 & 6 & 6 & 7 \\
\hline
\end{tabular}

From the analysis results of Table 2, it can be seen that both the AIM and the OPFM can get the nonlinearity and ensure the precision, in which the AIM give more adequate precision while the OPFM give more accurate precision. Besides, the nonlinearity of the function is increased because of the nonlinear of the Rosenblatt transformation.

TABLE III. THE ANALYSIS TIMES OF UNIVARIATE FUNCTION

\begin{tabular}{llllll}
\hline$k$ & & 3 & 4 & 5 & 6 \\
\hline \multirow{2}{*}{ Standard normal } & AIM & 13 & 19 & 31 & 27 \\
& OPFM & 7 & 7 & 7 & 7 \\
Lognormal & AIM & 19 & 19 & 19 & 27 \\
\multirow{2}{*}{ Extreme Type I } & OPFM & 7 & 7 & 7 & 18 \\
& AIM & 19 & 27 & 27 & 37 \\
& OPFM & 7 & 7 & 7 & 18 \\
\hline
\end{tabular}

And from table 3, one can see that the OPFM determining the nonlinearity of the univariate function needs lesser analysis times and has the higher efficiency. It is worth to point out that when the function does not only contain odd terms, due to the nonlinearity degree can be deduced via mean value estimate, the efficiency of AIM will be improved.

\section{CONCLUSIONS}

Under the premise of ensuring the precision, two methods have been proposed in this paper, i.e., adaptive iterative method (AIM) and orthogonal polynomial fitting method(OPFM). The numerical example shows that two methods can give satisfactory precision, both methods can determine the nonlinearity of univariate function and the number of nodes for each variable rationally. Comparatively speaking, orthogonal polynomial fitting method is more efficient than the adaptive iterative method, and the former has more practical value because it just requires the integration of low order moment.

\section{ACKNOWLEDGMENT}

The research reported in this paper was conducted with the support of the National Natural Science Foundation of China (Grant No. 51678092) and Construction and Technology Program of Chongqing, China (Grant No. 2016-0179).

\section{REFERENCES}

[1] Rosenblueth, E. 1975. Point estimates for probability moments. Proceedings of the National Academy of Sciences 72(10): 3812-3814.

[2] Rosenblueth, E. 1981. Two-point estimates in probability. Applied Mathematical Modelling 5(5): 329-335.

[3] Harr, ME. 1989. Probabilistic estimates for multivariate analyses. Applied Mathematical Modelling 13(5): 313-318.

[4] Chang, C.H et al. 1995. Evaluation of probability point estimate methods. Applied Mathematical Modelling 19(2): 95-105.

[5] Li, K.S. 1992. Point-estimate method for calculating statistical moments. Journal of Engineering Mechanics 118(7): 1506-1511.

[6] Zhao, Y.G. \& Ono, T. 2000. New point-estimates for probability moments. Journal of Engineering Mechanics 126(4): 433-436.

[7] Zhao, Y.G. \& Ono, T. 2001. Moment methods for structural reliability. Structural Safety 23(1): 47-75.

[8] Li, HS et al. 2008. Nataf transformation based point estimate method. Chinese Science Bulletin 53(17):2586-2592. 